\title{
Comparison between SARS-CoV and SARS-CoV 2
}

\author{
Meng Fang $1 *$ \\ ${ }^{1}$ University of Michigan, Ann Arbor 48104, USA
}

\begin{abstract}
COVID-19, which is officially called SARS-CoV-2, is a newly emerging viral respiratory illness leading to a global epidemic, which causes concerns among the global community in November 2019. SARS-CoV-2 is considered as the third global coronavirus epidemic in the past 20 years after SARS-CoV in 2002 and MERS in 2012. SARS is a viral respiratory illness caused by coronavirus SARS-CoV which was first reported in Guangdong, China in 2002. SARS-CoV-2 and SARS-CoV share similar and different biological features, clinical manifestations, region distribution, transmission mechanisms, and clinical treatments. In this paper, differences and similarities between SARS-CoV-2 and SARS-CoV are analyzed to provide valuable information for further research. Through analysis, we found SARS-CoV-2 and SARS$\mathrm{CoV}$ share a lot of similarities, but also have differences in clinical manifestations, pathogenicity, transmission rate and treatments.
\end{abstract}

\section{Introduction}

In November 2019, SARS-CoV-2 was found in a Seafood Wholesale Market in Wuhan, China, and now the infected cases have been reported all over the world. SARS-CoV-2 has been considered as the third respiratory illness caused by coronavirus after SARS$\mathrm{CoV}$ and MERS. The three coronaviruses are closely related, especially for SARS-CoV and SARS-CoV-2. However, differences remain between SARS-CoV and SARS-CoV-2 . In this paper the review of the two viruses on their similarities and differences in clinical manifestation, pathogenicity, transmission mechanism and treatments is provided. By analyzing their differences and similarities, valuable information on finding the pathogenicity, clinical feature and treatment of SARS-CoV-2 will be obtained.

\section{Clinical Manifestations}

SARS-CoV-2 has typical clinical symptoms of viral respiratory illness such as fever, cough, and shortness of breath [1]. Some uncommon symptoms of SARS-CoV-2 also include dizziness, confusion, chest pain, and diarrhea. SARS-CoV-2 can be diagnosed by using RTPCR, rPT-PCR, RT-LAMP, and radiology technology like CT scans.

\subsection{Symptoms}

Among the very first 41 patients discovered in Wuhan, the data indicate that common symptoms are fever, dry cough, myalgia, and fatigue. SARS-CoV shares common symptoms like fever, cough with SARS-CoV-2.
However, around $16 \%$ of SARS-CoV-2 patients showed symptoms of diarrhoea and around $23 \%$ to $70 \%$ percent of SARS patients showed symptoms of diarrhoea. Furthermore, up to $20 \%$ to $35 \%$ percent of SARS-CoV patients showed symptoms like vomiting while only $6 \%$ of the SARS-CoV-2 patients showed such symptoms [2]. SARS-CoV-2 patients also showed less probability of having symptoms like rhinorrhoea, sneezing, and sore throat, and other upper respiratory tracts, which suggests that SARS-CoV-2 is likely to infect the lower respiratory tract in the human body [1]. SARS-CoV-2 also has a lower fatality rate of $2.3 \%$ comparing to the $9.5 \%$ fatality rate of SAR-COV. The majority of death of SARS-CoV-2 occurs in older patients and severe cases occur in middle-aged patients that have chronic diseases such as diabetes and hepatitis B [3].

\subsection{Infected Group}

The infected groups also differ between COVID 19 patients and SARS-CoV patients. It is interesting to see that the male to female ratio for SARS-CoV-2 is 2.7 to 1 , while for SARS-CoV the male to female ratio is $1: 1.5$ [4]. Besides, in Italy and Spain, the death of males is two times of the death of females. There are some speculations on why such differences between gender exist in the case of SARS-CoV-2. Studies found that there is a positive correlation between the expression of the receptor for SARS-CoV-2: Angiotensin-converting enzyme 2, known as ACE 2, and the infection of the disease. Based on investigations and RNA sequencing, Asian males have higher expression of ACE 2 comparing to females, which may be a possible reason for higher transmission for males [5]. Also, gender difference causes a difference in inhibitory CD200

*Corresponding author: fmmeng@umich.edu 
receptors, which also plays a role in infection of SARSCoV-2. The in vivo study conducted by Karnam indicated that the lack of CD200R signaling improves type 1 interferon production and viral clearance, and it improves the condition of infection of another type of coronavirus known as Mouse Hepatitis coronavirus.

Such a phenomenon is found particularly in female mice, which further provides the assumption that gender causes a difference in the immune response to SARSCoV-2. The daily habits of males and females can also be a factor, as more males smoke and drink alcohol, which also can lead to a higher infection rate.

Table 1. Clinical characteristics of SARS-CoV-2, SARS and MERS [2]

\begin{tabular}{|c|c|c|}
\hline Cemographic General characteristics, \% of \\
cases & $\begin{array}{c}\text { COVID } \\
\text { Male }\end{array}$ & SARS \\
\hline Female & $40-60$ & $38-42$ \\
\hline Cardiovascular disease & $40-55$ & $64-68$ \\
\hline Chromic lung disease & $10-46$ & 8 \\
\hline Diabetes & $1-2$ & $1-2$ \\
\hline Malignancy & 10 & 16 \\
\hline Fever & $2-4$ & 6 \\
\hline Cough & $81-91$ & $99-100$ \\
\hline Dyspnoea & $48-68$ & $57-75$ \\
\hline Sore throat & $19-31$ & $40-42$ \\
\hline Dizziness and confusion & 29 & $13-25$ \\
\hline Diarrhoea & 22 & $4-43$ \\
\hline Nausea and vomiting & 16 & $23-70$ \\
\hline
\end{tabular}

\section{Pathogenicity}

Based on genetic analysis, SARS-CoV-2 and SARS$\mathrm{CoV}$ are genetically similar. There are 6 different regions in the genome sequences between SARS-CoV-2 and SARS-CoV, and the genome sequence of RNAdependent RNA polymerase (RdRp) is different for both viruses, which indicate that COVID-109 belongs to an independent subclade in the $\beta$-coronavirus genus.

SARS-CoV-2 was firstly discovered in Wuhan, China in 2019, and the very first cases of the virus were linked to Huanan Seafood Wholesale Market, where wild animals were sold. SARS-CoV-2 was found to belong to the Betacoronavirus genera of the family of Coronaviridae, which also includes SARS-CoV. According to gene sequences of the two viruses, SARSCoV-2 shares about $80 \%$ genetic similarities with SARS-CoV. SARS-CoV-2 is $88.8 \%$ identical to two batderived viruses. Further studies prove that SARS-CoV-2 shows 96.7\% similarity with BatCoV RaTG13, a coronavirus originated in Rhinolophus affinis, and sampled in Yunnan Province, China which indicates that the possible natural reservoir of SARS-CoV-2 is bats [1]. The possible intermediate host for SARS-CoV-2 is currently unknown as research, which indicates there is more than one intermediate host such as pangolin, minks, and snakes. SARS originated in Guangdong, China in 2002, and its natural reservoir is also bats. It was proved that the intermediate host for SARS-CoV is the civet cats and the civet cats in the wildlife markets directly transmitted SARS-CoV to humans.

Based on genetic evidences, SARS-CoV-2 and SARS-CoV are genetically similar. There are 6 different regions in the genome sequences between SARS-CoV-2 and SARS-CoV, and the genome sequence of RNAdependent RNA polymerase ( $\mathrm{RdRp}$ ) is different for both viruses, which indicate that COVID-109 belongs to an independent subclade in the $\beta$-coronavirus genus.

Both SARS-CoV-2 and SARS-CoV are zoonosis diseases, while SARS-CoV-2 is a recombinant virus between the bat virus and the source-unknown coronavirus. They share the same human cell receptor: the angiotensin-converting enzyme 2 (ACE2). ACE 2 is located at the plasma membrane of the tissue cells, especially for tissues at the lower respiratory tract, heart, kidney, and gastrointestinal tract [2]. The entry of the virus is done by attaching $\mathrm{S}$ glycoprotein (Spike Protein) to the ACE2 receptor at the cell membrane [6]. The mutation in the receptor binding domain (RBD) of the $\mathrm{S}$ protein increased pathogenicity of SARS-CoV by strengthening the affinity between the virus and the receptor [2]. For the SARS-CoV-2 virus, there is no mutation found in RBD that interacts with the ACE 2 
receptor, but mutations are found in other parts of RBD [2]. Overall, the amino acids in RBD are similar for SARS-CoV and SARS-CoV-2. Study shows that most amino acids in RBD of the $\mathrm{S}$ protein are similar for SARS-CoV and SARS-CoV-2.

The current data already showed that SARS-CoV-2 has a higher transmission rate than SARS-CoV, and analysis of the ACE 2 receptor affinity shows that SARS-CoV-2 can bind about 10 to 20 times more efficiently to ACE 2 receptor comparing to SARS-CoV [2]. Studies have already shown that there is a positive relationship between the expression of ACE 2 and the infection of SARS-CoV, and a study by Lu et al. has concluded a similar receptor binding protein between SARS-CoV and SARS-CoV-2. This further indicates higher efficiency of binding ACE 2 receptors might be a reason why SARS-CoV-2 has a higher transmission rate. There has been evidence suggested that there is a higher number of ACE 2 receptors in males compared to females, and the number also differs from ethnicity [4]. Asians, especially Asian males will have a higher number of ACE 2 receptors. Antibodies targeting ACE 2 will be important in treating the disease. Furthermore, research also suggests that the higher infectivity of SARS-CoV-2 is related to the special features of its $\mathrm{S}$ protein. The $\mathrm{S}$ protein is made up of 2 subunits $\mathrm{S} 1$ and $\mathrm{S} 2$, where $\mathrm{S} 1$ contains an $\mathrm{N}$ terminal domain connected to the RBD and S2 helps membrane fusion. For SARS$\mathrm{CoV}-2$, a furin cleavage site insertion appears at S1-S2 conjunction, and this feature doesn't appear in SARS$\mathrm{CoV}$ [7]. The presence of this site also contributes to a higher pathogenicity of SARS-CoV-2 comparing to SARS-CoV [6].

\section{Region Distribution and Transmission}

According to WHO, the regions having the highest amount of cases of SARS-CoV are China, China Hong Kong Special Administration, Taiwan, and Singapore. The region is concentrated in Asia with fewer other regions included. The total number of cases is 8422 with 916 deaths all over the world.

Based on Johns Hopkins Coronavirus Resource Center, the current number of cases of SARS-CoV-2 is $42,718,658$ cases with $1,150,614$ global death up to October 25. SARS-CoV-2 is spread all over the continents. Currently, the United States, India, and Brazil are among the countries that have the highest number of cases discovered; Europe and North America are the regions where most cases concentrate. SARSCoV-2 seems to have a higher transmission rate than SARS-CoV, and more regions are affected.

SARS-CoV-2 exhibits animal to human transmission and human to human transmission. The initial cases of SARS-CoV-2 suggest that the transmission was introduced from an unknown location while accelerated in the market through human to human transmission [1]. SARS-CoV also exhibits animal to human transmission and human to human transmission. SARS-CoV-2 seems to have a higher viral persistence comparing to SARS$\mathrm{CoV}$ as it can stay up to 9 days on the surface while SARS-CoV can only persist for 96 hours [1].

$\mathrm{R} 0$, which indicates the reproduction number and the average number of people who will get a contagious disease from a person who already had the disease, is also different between SARS-CoV and SARS-CoV-2. Estimated by WHO, the average R0 for SARS-CoV-2 is 2 to 2.5 , which is higher than SARS ranging from 1.7 to 1.9 , which gives another evidence for higher infectivity for SARS-CoV-2 [2].

Table 2. Main aspects of coronavirus disease 2019 (COVID-19) and severe acute respiratory syndrome (SARS)[4]

\begin{tabular}{|lll|}
\hline & COVID-19 & SARS \\
\hline Location of first detection & Wuhan, China & Guangdong, China \\
\hline Start date & December 2019 & November 2002 \\
\hline Incubation period & $2-10$ years (mean of 4-5 days) & $1-14$ years (mean of 5.1 days) \\
\hline Global cumulative incidence & 512701 cases (to date) & 8422 cases \\
\hline Deaths & 23595 (to date) & 916 \\
\hline Mortality & $4.6 \%$ & $10.8 \%$ \\
\hline Median age & 59 years (range of 15 to 89 years) & 35 years (range of 0 to 92 years) \\
\hline Male to female ratio & $2.7: 1$ & $1: 1.25$ \\
\hline Possible natural reservoir & Bat & Bat \\
\hline Possible intermediate host & Pangolins & Civet cats \\
\hline Ro & 1.4 to 2.5 (median of 1.95) & 3 \\
\hline Intestinal Symptoms & rare & $20 \%-25 \%$ of cases \\
\hline Predominant cellular receptor & ACE2 & ACE2 \\
\hline
\end{tabular}

Abbreviation: $\mathrm{ACE}=$ Angiotensin-converting enzyme.

\section{Treatments}

Up to today, there is no particular treatment for SARSCoV-2. People learned lessons from previous coronavirus outbreak such as SARS-CoV and similar approaches are also adopted for treating SARS-CoV-2.

Neutralizing antibodies have been effective in preventing viral infection in human bodies, and this method has been used during the 2003 SARS-CoV pandemic. Based on the biological features of the 
coronavirus, researchers targeted the binding region of the $\mathrm{S}$ protein of the virus and the receptors to develop antibodies against the living SARS-CoV virus.

Nowadays, specific antiviral treatments for SARSCoV-2 remain unclear. Isolation of patients, hormone treatments, antiviral and symptomatic treatments are still be utilized to treat SARS-CoV-2 [3]. Some treatments for SARS-CoV were proved to have clinical benefits SARS-CoV-2. For example, Lopinavir/Ritonavir, a protease inhibitor, has lowered the risk of getting acute respiratory distress syndrome (ASDR) or death caused by SARS-CoV-2. Interferon and hormones also showed effects: interferon $\alpha 2 \mathrm{~b}$ demonstrated effects in the lab to treat SARS-CoV-2.

TCM (Traditional Chinese Medicine) was also found useful in decreasing the symptoms of SARS-CoV-2. For instance, acupuncture and moxibustion therapy are used to prevent the transmission of the disease and the treatment of the disease, and the Shufeng Jiedu Capsule (SFJDC), a traditional Chinese medicine capsule are also found to be useful [8].

New effective treatments are also under development. Joint research at Shanghai Institute of Materia Medica, Chinese Academy of Medicines, and Shanghai Tech University has given out 30 potential medicines that are found effective in treating SARS-CoV-2 [3]. What's more, research in the US had found that intravenous remdesivir prevents adverse reactions during the treatment, and ongoing research had started in Wuhan and Beijing in China [3].

\section{Conclusion}

In Conclusion, SARS-CoV and SARS-CoV-2 share homologous features. They are highly genetically related, and they share similar outbreak origins. They share similar clinical manifestations of viral respiratory illness, pathogenetic features like ACE2 receptor, transmission mechanisms like the human to human and the animal to human transmissions, and similar clinical treatments.

However, they also have differences in various aspects. SARS-CoV-2 is more related to a coronavirus originated in Rhinolophus affinis comparing to SARSCoV-2, indicating some parts of genetic differences. In general, SARS-CoV-2 has a higher male to female patient ratio, showing a higher binding ability to ACE2 receptors, a higher transmission rate, and a higher reproductive ability comparing to SARS-CoV. These differences will be the most valuable information for the researches in the future discovery of the treatment for COVID-19 and maybe the breakthrough of some unsolved problem of pandemic today.

\section{Acknowledgement}

I would like to express my special thanks of gratitude to Professor Zhibin Wang at Johns Hopkins University, who provided me with guidance in writing this thesis as well as my professors at University of Michigan, who also provided me with valuable academic resources and information in completing this thesis.

\section{References}

1. Harapan, H., Itoh, N., Yufika, A., Winardi, W., Keam, S., Te, H., Megawati, D., Hayati, Z., Wagner, A. L., \& Mudatsir, M. (2020). Coronavirus disease 2019 (SARS-COV-2): A literature review. Journal of infection and public health, 13(5), 667-673. https://doi.org/10.1016/j.jiph.2020.03.019

2. Petrosillo, N., Viceconte, G., Ergonul, O., Ippolito, G., \& Petersen, E. (2020). SARS-COV-2, SARS and MERS: are they closely related?. Clinical microbiology and infection : the official publication of the European Society of Clinical Microbiology and Infectious Diseases, 26(6), 729-734. https://doi.org/10.1016/j.cmi.2020.03.026

3. Xu, J., Zhao, S., Teng, T., Abdalla, A. E., Zhu, W., Xie, L., Wang, Y., \& Guo, X. (2020). Systematic Comparison of Two Animal-to-Human Transmitted Human Coronaviruses: SARS-CoV-2 and SARSCoV. Viruses, 12(2), 244. https://doi.org/10.3390/v12020244

4. Caldaria, A., Conforti, C., Di Meo, N., Dianzani, C., Jafferany, M., Lotti, T., Zalaudek, I., \& Giuffrida, R. (2020). SARS-COV-2 and SARS: Differences and similarities. Dermatologic therapy, e13395.

Advance online publication. https://doi.org/10.1111/dth.13395

5. Bwire G. M. (2020). Coronavirus: Why Men are More Vulnerable to SARS-COV-2 Than Women?. SN comprehensive clinical medicine, 1-3. Advance online publication. https://doi.org/10.1007/s42399-020-00341-w

6. Fani, M., Teimoori, A., \& Ghafari, S. (2020). Comparison of the COVID-2019 (SARS-CoV-2) pathogenesis with SARS-CoV and MERS-CoV infections. Future Virology, 10.2217/fvl-2020-0050. https://doi.org/10.2217/fvl-2020-0050

7. Sironi, M., Hasnain, S. E., Rosenthal, B., Phan, T., Luciani, F., Shaw, M. A., Sallum, M. A., Mirhashemi, M. E., Morand, S., González-Candelas, F., \& Editors of Infection, Genetics and Evolution (2020). SARS-CoV-2 and SARS-COV-2: A genetic, epidemiological, and evolutionary perspective. Infection, genetics and evolution : journal of molecular epidemiology and evolutionary genetics in infectious diseases, 84, 104384. https://doi.org/10.1016/j.meegid.2020.10438

8. Zhao, Z., Li, Y., Zhou, L., Zhou, X., Xie, B., Zhang, W., \& Sun, J. (2020). Prevention and treatment of COVID-19 using Traditional Chinese Medicine: A review. Phytomedicine: international journal of phytotherapy and phytopharmacology, 153308. Advance online publication. https://doi.org/10.1016/j.phymed.2020.153308 